\title{
A SEARCH FOR WOLF-RAYET STARS IN NGC 300
}

\author{
M. AZZOPARDI ${ }^{1,2}$, G. MURATORIO ${ }^{1}$, J. BREYSACHER ${ }^{3}$ and \\ G. TESTOR ${ }^{4}$ \\ ${ }^{1}$ Observatoire de Marseille, F-13248 Marseille Cedex 04, France \\ ${ }^{2}$ Canada-France-Hawaii Telescope, P.O. Box 1597, Kamuela HI 96743, U.S.A. \\ ${ }^{3}$ European Southern Observatory, D-85748 Garching, B.R. Deutschland \\ ${ }^{4}$ DAEC, Observatoire de Meudon, F-92195 Meudon Cedex, France
}

Abstract. Preliminary results of our NGC 300 Wolf-Rayet star survey are presented.

Key words: stars: Wolf-Rayet - galaxies: NGC300 - surveys - techniques: spectroscopy

As to the present state of the detection of the Wolf-Rayet (WR) stars in nearby galaxies (see Massey \& Armandroff 1991), NGC 300 - which is considered as a twin of M33 - is obviously a bona fide candidate for a rather complete survey for WR stars, due to its relatively close distance in the Sculptor group of galaxies and to its favourable, almost face-on orientation. Consequently, with the purpose of extending to other nearby galaxies the surveys for WR stars performed in the Local group galaxies we have carried out observations of NGC $300\left((M-m)_{0} \approx 26\right)$ with the ESO New Technology Telescope (NTT), using the Multimode Instrument (EMMI).

For this purpose we introduced an observing technique which turned out to be very efficient for the detection of the WR stars in the Magellanic Clouds (see Azzopardi \& Breysacher 1980, and papers by the same authors quoted therein) as well as to a certain extent in NGC 6822 (Westerlund et al. 1983). We used EMMI in the slitless spectroscopy mode equipped with a grism providing $515 \AA / \mathrm{mm}$ dispersion ( $9.7 \AA / \mathrm{px}, 19 \mu \mathrm{m} \mathrm{px}$ ) in combination with a $145 \AA$ bandwidth interference filter with a peak wavelength at 4692 $\AA$. In that spectral domain WR stars show up strongly due to the emission mainly from either $\lambda 4650 \mathrm{CIII}$ (WC type) or $\lambda 4686 \mathrm{He}$ II (WN type). By reducing both the sky background and the length of the spectra on the detector the interference filter allows us to reach very faint stars even in very crowded NGC 300 regions, apart in the giant $\mathrm{H}$ II regions.

Up to now the survey of 6 fields $7.6 \times 7.6$ arcmin $^{2}$ wide $(3$ exposures of $3600 \mathrm{~s}$ each) covering approximately $70 \%$ of the central regions of NGC 300 - using a semi-automatic procedure to extract and reduce slitless spectra (Muratorio \& Azzopardi 1994) - resulted in the identification of about 80 possible WR star candidates. We found again all the WR stars actually spectroscopically confirmed by Schild \& Testor (1991, 1992). Subsequent multi-object spectroscopy (MOS) with EMMI of a sample of the newly discovered WR star candidates, using a grism with a dispersion of $4.6 \AA / \mathrm{px}$ 


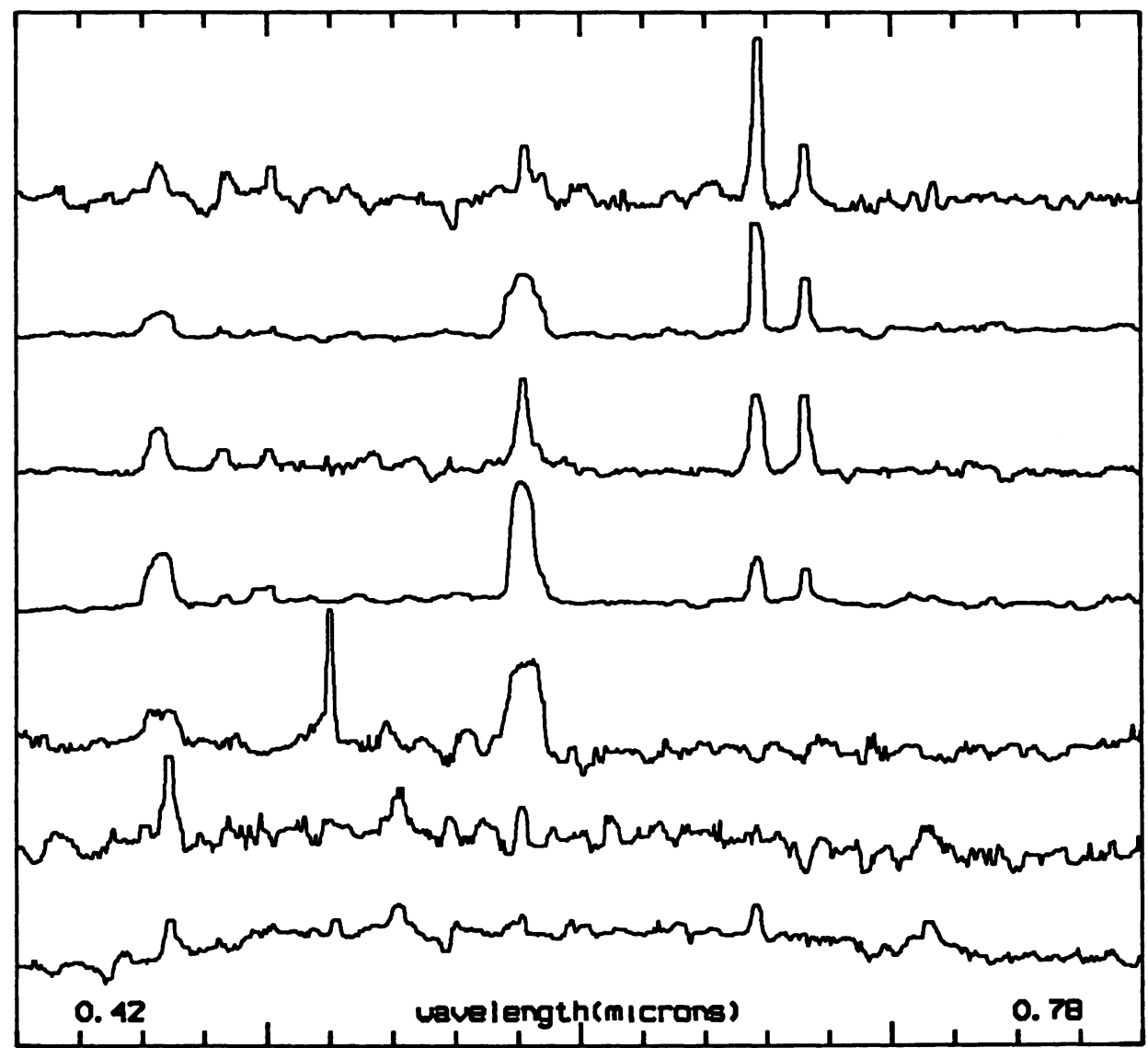

Fig. 1. Spectrograms $(246 \AA / \mathrm{mm})$ of some newly identified WR stars in NGC 300

(19 $\mu \mathrm{m} \mathrm{px}$ ), confirmed the WR star nature of 14 objects, increasing to 24 the total number of WR stars known at the present time in NGC 300.

\section{References}

Azzopardi, M., Breysacher, J. 1980, A 6 A Suppl. 39, 19

Massey, P., Armandroff, T.E. 1991, in: K.A. van der Hucht \& B. Hidayat (eds.), WolfRayet Stars and Interrelations with Other Massive Stars in Galaxies, Proc. IAU Symp. No. 143 (Dordrecht: Kluwer), p. 575

Muratorio, G., Azzopardi, M. 1994, in: H.T. MacGillavry (ed.), Wide-Field Imaging in Astronomy, Proc. IAU Symp. No. 161 (Dordrecht: Kluwer), in press

Schild H., Testor G. 1991, $A$ E $A$ 243, 115

Schild H., Testor G. 1992, A E A 266, 145

Westerlund, B.E., Azzopardi, M., Breysacher, J., Lequeux, J. 1983, A\&A 123, 159 